\title{
Long non-coding RNA SChLAP1 regulates the proliferation of triple negative breast cancer cells via the miR-524-5p/HMGA2 axis
}

\author{
XIANGDONG BAI $^{1}$, SHENGXIAO ZHANG ${ }^{2}$, JUN QIAO $^{2}$, XIAOLONG XING $^{3}$, \\ WEINA $\mathrm{LI}^{4}$, HUANHU ZHANG ${ }^{5}$ and $\mathrm{JUN} \mathrm{XIE}^{6}$
}

\begin{abstract}
${ }^{1}$ Department of Breast Surgery, Shanxi Provincial Cancer Hospital, Shanxi Medical University, Taiyuan, Shanxi 030013;
${ }^{2}$ Department of Rheumatism and Immunology, Second Clinical Medical College, Shanxi Medical University;

${ }^{3}$ Research and Development Division, Shanxi Immune Medical Technology Co., Ltd.; ${ }^{4}$ Department of Radiotherapy, Shanxi Provincial Cancer Hospital, Shanxi Medical University, Taiyuan, Shanxi 030001; ${ }^{5}$ Department of Gastroenterology, Shanxi Provincial Cancer Hospital, Shanxi Medical University, Taiyuan, Shanxi 030013; ${ }^{6}$ Department of Biochemistry and Molecular Biology, Shanxi Provincial Cancer Hospital, Shanxi Medical University, Taiyuan, Shanxi 030001, P.R. China
\end{abstract}

Received August 16, 2020; Accepted February 10, 2021

DOI: $10.3892 / \mathrm{mmr} .2021 .12085$

\begin{abstract}
Long non-coding RNA (lncRNA) second chromosome locus associated with prostate-1 (SChLAP1), also named LINC00913, has been reported to accelerate the carcinogenesis of prostate cancer. The aim of this study was to explore the role and mechanism of SChLAP1 in triple negative breast cancer (TNBC). The expression of SChLAP1 in TNBC tissues and cells was determined by reverse transcription quantitative PCR. The effects of SChLAP1 on the growth of TNBC cells was evaluated by detecting cell viability, colony formation and apoptosis. The present study determined that SChLAP1 was upregulated in TNBC tissues and was associated with the long-distant lymph node metastasis of patients with TNBC. Knockdown of SChLAP1 significantly inhibited cell viability and colony formation, and triggered apoptosis of TNBC cells. Bioinformatics analysis suggested that SChLAP1 acted as a sponge of microRNA (miR)-524-5p and negatively modulated the expression of miR-524-5p. An inverse correlation was also identified between the expression levels of SChLAP1 and miR-524-5p in TNBC tissues. Furthermore, the results
\end{abstract}

Correspondence to: Dr Huanhu Zhang, Department of Gastroenterology, Shanxi Provincial Cancer Hospital, Shanxi Medical University, 3 New Worker's Road, Xinghualing, Taiyuan, Shanxi 030013, P.R. China

E-mail: zhh349@126.com

Dr Jun Xie, Department of Biochemistry and Molecular Biology, Shanxi Provincial Cancer Hospital, Shanxi Medical University, 56 Xinjian South Road, Yingze, Taiyuan, Shanxi 030001, P.R. China E-mail: xiejun349@126.com

Key words: triple negative breast cancer, second chromosome locus associated with prostate-1, microRNA-524-5p, High Mobility Group AT-Hook 2 demonstrated that SChLAP1 interacted with miR-524-5p, and subsequently regulated the expression level of High Mobility Group AT-Hook 2 (HMGA2) in TNBC cells. It was also found that the overexpression of HMGA2 rescued the suppressed viability of TNBC cells induced by SChLAP1 knockdown. In conclusion, the present findings demonstrated that SChLAP1 modulated the malignant tumor behaviors of TNBC cells by regulating HMGA2 and subsequently restraining miR-524-5p.

\section{Introduction}

Triple negative breast cancer (TNBC) is characterized by the absence of estrogen and progesterone receptors and Erb-b2 receptor tyrosine kinase 2 , and this type of cancer has been the second leading cause of cancer-associated mortalities among women (1-3). Although significant progression has been achieved in the therapy of breast cancer, chemotherapy remains the primary treatment option for patients with TNBC (4). Therefore, the clinical outcome of patients with TNBC is relatively worse compared with that of other types of breast cancer due to the inefficiency of endocrine therapy and targeted therapy (4). As the metastatic risk of TNBC is high, it is important to elucidate the molecular mechanism underlying the progression of TNBC and identify available therapeutic targets.

Long non-coding RNAs (lncRNAs) are a class of non-coding transcripts $>200$ nucleotides in length, which have a wide range of regulatory functions in both physiological and pathological processes $(5,6)$. As another set of non-coding RNAs, microRNAs (miRNAs/miRs) are defined as single-stranded, small RNAs involved in post-transcriptional regulation of gene expression via interacting with the 3'-untranslated region (UTR) of target mRNAs (7-10). Interestingly, accumulating evidence has suggested that lncRNAs and miRNAs participate in the tumorigenesis of TNBC by modulating the expression of oncogenes or tumor suppressors (11-15). One functional mechanism consists of 
lncRNAs serving as competing endogenous RNAs (ceRNAs) for miRNAs to regulate miRNA and miRNA-targeted mRNAs (15). For example, lncRNA antisense RNA in the INK4 locus acted as a ceRNA of miR-199a and promoted the progression of TNBC (16). Moreover, a recent study revealed that lncRNA LINC00173 enhanced the development of TNBC by sponging miR-490-3p (17). These findings indicate that targeting lncRNAs may be promising to improve the positive outcomes of TNBC.

Second chromosome locus associated with prostate-1 (SChLAP1), also named LINC00913, is an upregulated IncRNA that is proven to participate in oncogenesis in prostate cancer (18-20). SChLAP1 accelerates the proliferation and metastasis of prostate cancer via sponging miR-198, and subsequently modulates the MAPK1 signaling pathway (20). Highly expressed SChLAP1 is a possible tissue-based biomarker for identifying patients with prostate cancer at higher risk of lethal progression (18). Additionally, silencing SChLAP1 suppresses the malignant phenotype of bladder cancer cells (21). This evidence indicates that abnormal expression of SChLAP1 occurs in cancer, and interrupting SChLAP1 may be a therapeutic solution for patients with cancer. However, the expression level and functional mechanism of SChLAP1 in TNBC have not been fully elucidated.

The aim of the present study was to investigate the clinical significance and biological function of SChLAP1, as well as elucidate the associated miRNAs that mediate the regulatory role of SChLAP1 in the malignancy of TNBC.

\section{Materials and methods}

Patients and tissues samples. A total of 50 paired TNBC tissues and matched adjacent normal tissues $(\sim 2 \mathrm{~cm}$ from the margin of the TNBC tissues) were obtained from the Shanxi Provincial Cancer Hospital between January 2012 and September 2014. Tissues were immediately frozen in liquid nitrogen and stored at $-80^{\circ} \mathrm{C}$. None of the patients received radiotherapy or chemotherapy before the surgery. This study was performed in accordance with the Declaration of Helsinki and was approved by the Ethics Committee of Shanxi Provincial Cancer Hospital (approval no. 0425245-1). Written informed consent statements were provided by all the enrolled patients. The clinical data of the patients enrolled in this study are presented in Table SI.

Cell culture and transfection. Human TNBC cell lines, including MDA-MB-231, SKBR3, BT-549 and HCC-1937, and the normal breast epithelial cell line MCF10A were purchased from the American Type Culture Collection. Cells were cultured in RPMI-1640 medium (Sigma-Aldrich; Merck KGaA), supplemented with 10\% FBS (Invitrogen; Thermo Fisher Scientific, Inc.) in a humidified atmosphere with $5 \% \mathrm{CO}_{2}$ at $37^{\circ} \mathrm{C}$.

The small interference RNA (siRNA) pool (two guaranteed siRNA mixtures, cat. no. 4392420; Thermo Fisher Scientific, Inc.) targeting SChLAP1, the miR-524-5p mimic (5'-CUACAAAGGGAAGCACUUUCUC-3') and the relative controls (5'-GGUUCGUACGUACACUGUUCA-3') were obtained from Shanghai GenePharma Co., Ltd. Flag-tagged High Mobility Group AT-Hook 2 (HMGA2) plasmid (100 ng; cat. no. 25409) was purchased from Addgene, Inc. A total of $50 \mathrm{nM}$ siRNA or miRNA was transfected into the TNBC cells using Lipofectamine ${ }^{\circledR} 2000$ transfection reagent (Invitrogen; Thermo Fisher Scientific, Inc.) at room temperature for $15 \mathrm{~min}$. Cells were cultured for $48 \mathrm{~h}$ before further analysis.

$R N A$ extraction and reverse transcription quantitative PCR $(R T-q P C R)$ analysis. Total RNA was extracted from frozen tissues or cultured cells using TRIzol ${ }^{\circledR}$ reagent (Invitrogen; Thermo Fisher Scientific, Inc.) according to the manufacturer's instructions. For the detection of miRNAs, RT $\left(37^{\circ} \mathrm{C}\right.$ for $10 \mathrm{~min}, 85^{\circ} \mathrm{C}$ for $5 \mathrm{sec}$ and $4^{\circ} \mathrm{C}$ for $15 \mathrm{~min}$ ) was performed with the TaqMan miRNA RT kit (Takara Biotechnology Co., Ltd.). The relative expression level of miR-524-5p was compared with that of U6 RNA using the TaqMan Universal PCR Master mix (Applied Biosystems; Thermo Fisher Scientific, Inc.) according to the manufacturer's protocol. For lncRNA or mRNA analysis, cDNA was synthesized with the TaqMan High-Capacity Complementary DNA RT kit (Tiangen Biotech Co., Ltd.). Gene expression was detected using the TaqMan Fast PCR Master mix (Applied Biosystems; Thermo Fisher Scientific, Inc.) according to the manufacturer's instructions. The expression level of GAPDH was also examined as the internal control. The thermocycling conditions were as follows: Initial denaturation at $95^{\circ} \mathrm{C}$ for $5 \mathrm{~min}$, followed by 40 cycles of denaturation at $95^{\circ} \mathrm{C}$ for $15 \mathrm{sec}$, annealing at $60^{\circ} \mathrm{C}$ for $15 \mathrm{sec}$ and extension at $72^{\circ} \mathrm{C}$ for $15 \mathrm{sec}$. The primers used in this study were as follows: SChLAP1 forward, 5'-GGG AAGAAGTGCCAGATGCT-3' and reverse, 5'-CAGCTTCTT CAGGGAGGTGG-3'; miR-524-5p forward, 5'-GCTGTGACC CTACAAAGGGA-3' and reverse, 5'-AGCATCAACTTCAAC GCTGC-3'; U6 RNA forward, 5'-CTCGCTTCGGCAGCA CATATACT-3' and reverse, 5'-ACGCTTCACGAATTTGCG TGTC-3'; GADPH forward, 5'-CGGAGTCAACGGATTTGG TCGTAT-3' and reverse, 5'-AGCCTTCTCCATGGTGGT GAAGAC-3'; and HMGA2 forward, 5'-TTCAGCCCAGGG ACAACC-3' and reverse, 5'-GCTGCTTTAGAGGGACTC TTGT-3'. The relative expression of targets was quantified with the $2^{-\Delta \Delta \mathrm{Cq}}$ method (22). The experiments were performed in triplicate.

Cell Counting Kit-8 (CCK-8) assay. The viability of TNBC cells transfected with the indicated expressing vectors was determined using CCK-8 (Beyotime Institute of Biotechnology) following the manufacturer's instructions. Cells were seeded in a 96-well plate with 1,000 cells per well. After culturing overnight, $10 \mu$ l CCK- 8 reagent was added to the medium at the indicated time interval (1, 2, 3, 4 and 5 day), and the plates were incubated for additional $4 \mathrm{~h}$ at $37^{\circ} \mathrm{C}$. The absorbance of each well was measured at the wavelength of $450 \mathrm{~nm}$ with a microplate reader (EnSpire 2300; PerkinElmer, Inc.).

Bioinformatics analysis. The mRNA expression profiles of TNBC (Homo sapiens) were downloaded from the Gene Expression Omnibus (GEO; http://www.ncbi.nlm.nih. gov/geo/) database. GSE58135 (expression profiling via high throughput sequencing) was used to identify hub lncRNAs associated with TNBC. The dataset provided gene expression profiles of 16 TNBC primary tumors and 16 breast tissues adjacent to TNBC primary tumors (female patients, 39-64 years 
old). High throughput sequencing raw data (Fastq files) for GSE58135 were evaluated using FastQC (version 0.11.8, http://www.bioinformatics.babraham.ac.uk/projects/fastqc/). Prefiltering of the quality of reads was performed using trim_galore (version 0.6.4_dev, http://www.bioinformatics. babraham.ac.uk/projects/trim_galore/). Filtered reads were aligned using STAR for the 65,217 annotated genes obtained from the GRCh38 (v79) build of the human genome (23). The read counts per gene were quantified using the featureCounts (version 1.6.4) using annotations from the GRCh38 (v79) build of the human genome (24). The Bioconductor R package DESeq2 (version 1.26.0; http://www.bioconductor. org/packages/release/bioc/html/DESeq2.html) was used to identify the differentially expressed genes between patients with TNBC and controls in the raw expression counts, which were adjusted for library size. Genes were considered to be differentially expressed when the Benjamini-Hochberg adjusted P-value [false discovery rate (FDR)] was $<0.05$ and the $\log 2$ fold-change $(\mathrm{FC}) \geq 4$ between TNBC tissues and control groups.

Target prediction. The targeted miRNAs of SChLAP1 were predicted using the online software program miRDB (version 5.0; http://mirdb.org/) (25). The highest rank among all the candidates was assigned to miR-524-5p. The potential targets of miR-524-5p were identified using the TargetScan database (version 7.2; http://www.targetscan.org/). The 3'-UTR of HMGA2 was found to contain complementary binding sites of miR-524-5p.

Luciferase reporter assay. The wild-type (WT) or mutated (Mut) fragment of SChLAP1 containing the predicted binding sites of miR-524-5p was inserted into the pmirGLO reporter vector (Promega Corporation). TNBC cells were co-transfected with $50 \mathrm{nM}$ miR-524-5p mimic and $200 \mathrm{ng}$ WT or Mut luciferase plasmid of SChLAP1 using Lipofectamine ${ }^{\circledR} 2000$ (Invitrogen; Thermo Fisher Scientific, Inc.). The luciferase activity was measured after transfection for $48 \mathrm{~h}$ using the Dual-Luciferase reporter analysis kit (Promega Corporation) following the manufacturer's protocol. The activity of Renilla luciferase was detected to normalize the activity of firefly. The experiments were performed in three replicates.

Western blot analysis. TNBC cells were lysed with RIPA buffer (Beijing Solarbio Science \& Technology Co., Ltd.) containing protease inhibitor and subjected to BCA protein assay to evaluate the protein concentration. Next, $20 \mu \mathrm{g}$ protein lysates were loaded onto $15 \%$ SDS-PAGE gels for electrophoresis, and subsequently separated proteins were transferred onto PVDF membranes. The membranes were blocked with $5 \%$ non-fat milk for $1 \mathrm{~h}$ at room temperature and subsequently incubated with primary antibody against HMGA2 (1:1,000; cat. no. ab97276; Abcam) or GAPDH (1:3,000; cat. no. ab181602; Abcam) overnight at $4^{\circ} \mathrm{C}$. After washing three times with PBS with $0.1 \%$ Tween-20, the membrane was incubated with Goat Anti-Rabbit IgG H\&L (HRP) secondary antibody (1:5,000; cat. no. ab205718; Abcam) for $1 \mathrm{~h}$ at room temperature. The expression of GAPDH was detected as the loading control. Protein bands were visualized with an ECL kit (Pierce; Thermo Fisher Scientific, Inc.) following the manufacturer's instructions. The semi-quantification of the western blotting bands was performed using the ImageJ software (v1.8.0; National Institutes of Health).

Colony formation assay. TNBC cells were transfected with siRNA-Control or siRNA-SChLAP1 and seeded into 6-well plates with 500 cells per well. Cells were cultured for 2 weeks with RPMI-1640 medium containing 10\% FBS. Colonies were washed with cold PBS and fixed with $75 \%$ methanol (Beyotime Institute of Biotechnology) at room temperature for $10 \mathrm{~min}$. Then, colonies were stained with $0.1 \%$ crystal violet for $15 \mathrm{~min}$ at room temperature and counted with a light microscope (magnification, $\mathrm{x} 40$ ).

Cell apoptosis. Cells were transfected with siRNA-Control or siRNA-SChLAP1 for $48 \mathrm{~h}$. The apoptosis of cells (early and late apoptotic cells) was analyzed via flow cytometry (FACScalibur; BD Biosciences) using the Annexin V-FITC/PI kit (BD Biosciences). Cells were resuspended with the binding buffer and stained with the Annexin V-FITC at room temperature for 30 min followed by staining with PI in the dark for $10 \mathrm{~min}$ at room temperature. The cell apoptosis profile was analyzed using the CellQuest Software version 0.9.3.1 (BD Biosciences).

Statistical analysis. The data are presented as the mean \pm SD from three independent experiments. Statistical significance was determined with paired Student's t-test for data in Fig. 1B and C, or one-way ANOVA followed by Tukey's test using GraphPad software (version 7.0; GraphPad Software, Inc.). Differences between two groups were analyzed using an unpaired t-test. The correlation between miR-524-5p and SChLAP1 was assessed via the Pearson's test. $\mathrm{P}<0.05$ was considered to indicate a statistically significant difference.

\section{Results}

SChLAP1 expression is increased in TNBC tissues and cell lines. To determine which lncRNAs were aberrantly expressed in TNBC, bioinformatics analysis was conducted using the GEO (http://www.ncbi.nlm.nih.gov/geo/) database that covered the gene expression profiles obtained from 16 TNBC tissues and 16 adjacent non-cancerous tissues. It was found that lncRNA SChLAP1 was among the 10 most upregulated lncRNAs in TNBC tissues (Figs. 1A and S1).

To verify the increased expression of SChLAP1 in TNBC, the expression levels of SChLAP1 in a larger sample size $(n=50)$ of TNBC tissues and matched non-cancerous tissues were determined via RT-qPCR analysis. The results demonstrated that the expression of SChLAP1 was significantly upregulated in TNBC tissues compared with that of the adjacent normal tissues (Fig. 1B). In addition, patients with distant lymph node metastasis $(n=16)$ had a relative higher expression level of SChLAP1 compared with patients without metastasis $(n=34)$ (Fig. 1C). To further confirm the increased expression of SChLAP1 in TNBC, the expression level of SChLAP1 in TNBC cell lines was also determined. Significantly increased expression levels of SChLAP1 were found in TNBC cell lines compared with those of normal MCF10A cells (Fig. 1D). These findings indicated that the SChLAP1 was upregulated in TNBC. 
A

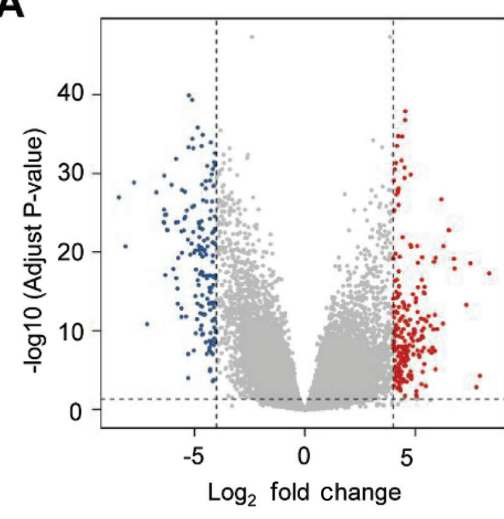

C

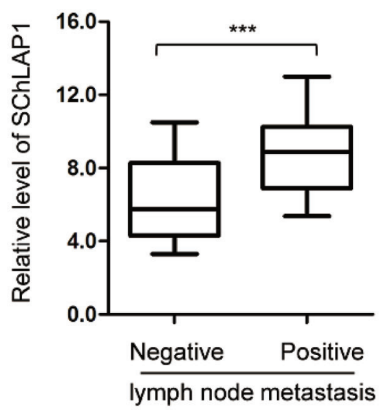

B

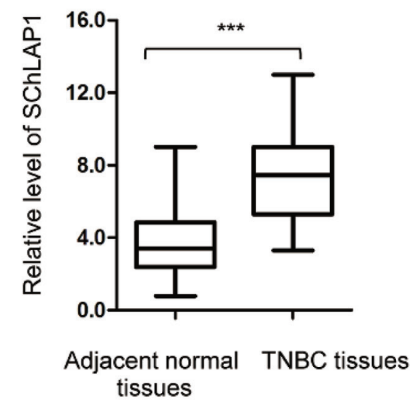

D

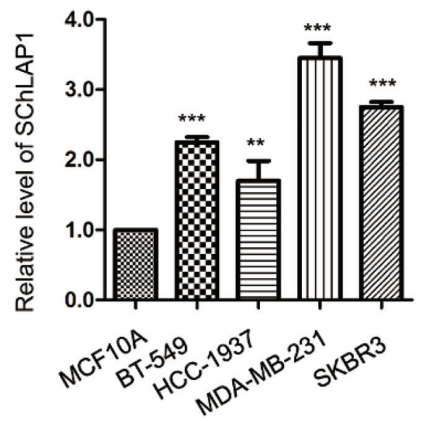

Figure 1. SChLAP1 is upregulated in TNBC tissues and cell lines. (A) Dot plots of upregulated, downregulated and non-significantly differentiated expressed long non-coding RNAs in 16 TNBC tissues and adjacent normal tissues. (B) SChLAP1 expression levels between TNBC tissues and paired non-cancerous normal tissues were detected via reverse transcription-quantitative PCR. (C) Expression levels of SChLAP1 in patients with TNBC with LNM were higher compared with those in patients without LNM. (D) Relative expression of SChLAP1 in normal cell MCF-10A and TNBC cell lines. ${ }^{* *} \mathrm{P}<0.01$, ${ }^{* * *} \mathrm{P}<0.001$ vs. control group. LNM, lymph node metastasis; TNBC, triple negative breast cancer; SChLAP1, second chromosome locus associated with prostate-1.

Knockdown of SChLAPI suppresses the viability and colony formation of TNBC cells, and also triggers apoptosis of TNBC cells. Given the upregulation of SChLAP1 in TNBC, the effects of SChLAP1 on the malignant behaviors of TNBC cells were examined via knockdown of the expression of SChLAP1. MDA-MB-231 and SKBR3 cells were transfected with siRNA-SChLAP1 or non-targeting control (siRNA-control), and the expression of SChLAP1 was validated via RT-qPCR. The results demonstrated that the expression of SChLAP1 was significantly decreased after the transfection of siRNA-SChLAP1 in TNBC cells (Fig. 2A).

The influence of SChLAP1 on the viability of TNBC cells was determined via the CCK-8 assay. As presented in Fig. 2B and C, compared with cells transfected with siRNA-control, knockdown of SChLAP1 significantly suppressed the proliferative capacity of both MDA-MB-231 and SKBR3 cells. Consistently, the colony formation analysis also indicated that knockdown of SChLAP1 resulted in the suppressed viability of TNBC cells (Fig. 2D).

To further validate the inhibitory effects on the malignant behaviors of TNBC cells induced by knockdown of SChLAP1, the apoptosis of both MDA-MB-231 and SKBR3 cells was evaluated using flow cytometry analysis. The results indicated that knockdown of SChLAP1 significantly increased the apoptotic percentage of TNBC cells compared with the control group (Fig. 2E). Collectively, these findings suggested that SChLAP1 may be essential in regulating the malignant phenotypes of TNBC cells.

SChLAP1 serves as a sponge of $m i R-524-5 p$. To examine the possible underlying mechanism via which SChLAP1 affected the activities of TNBC cells, the targeted miRNAs of SChLAP1 were predicted using the online tool (http://mirdb.org/). The bioinformatics analysis identified that miR-524-5p harbored the possible binding sites of SChLAP1 (Fig. 3A). To confirm the binding between SChLAP1 and miR-524-5p, a luciferase reporter assay was performed by co-transfecting luciferase vector carrying WT or Mut SChLAP1 and miR-524-5p mimic. As indicated in Fig. 3B and C, overexpression of miR-524-5p significantly decreased the luciferase activity of TNBC cells expressing WT but not Mut SChLAP1, which suggested that there was specific binding between SChLAP1 and miR-524-5p.

To investigate whether the binding affected the stability of miR-524-5p, the expression level of miR-524-5p in TNBC cells transfected with siRNA-SChLAP1 or siRNA-control was determined via RT-qPCR. The data demonstrated that knockdown of SChLAP1 significantly upregulated the expression level of miR-524-5p in both MDA-MB-231 and SKBR3 cells (Fig. 3D), which suggested that there was negative regulation of SChLAP1 upon the expression of miR-524-5p. To support this conclusion, the expression of miR-524-5p in TNBC tissues and matched non-cancerous tissues was detected, and it was determined that miR-524-5p was significantly downregulated in TNBC tissues 
A
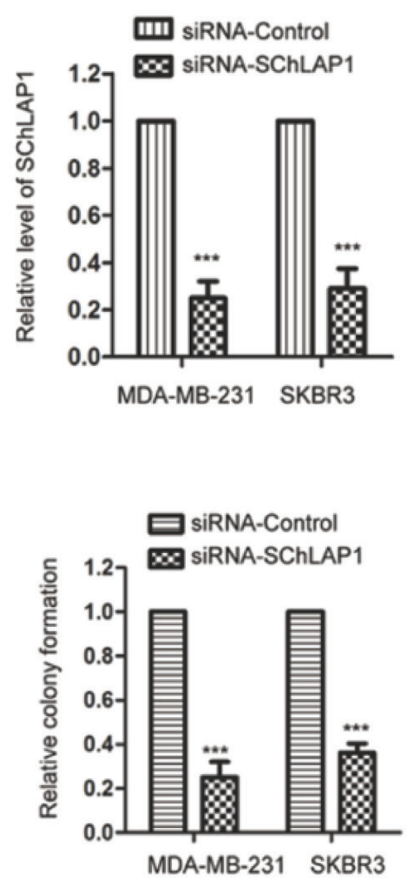

B

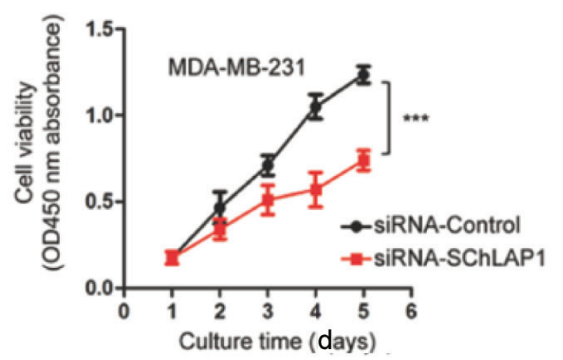

C

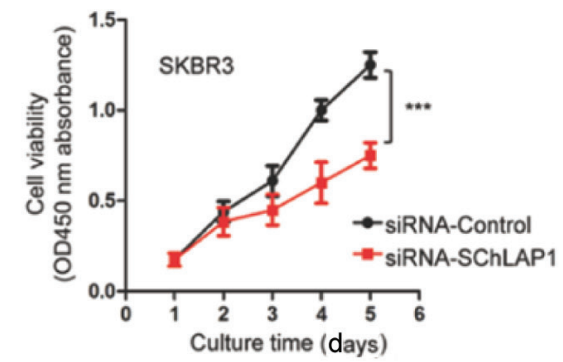

E
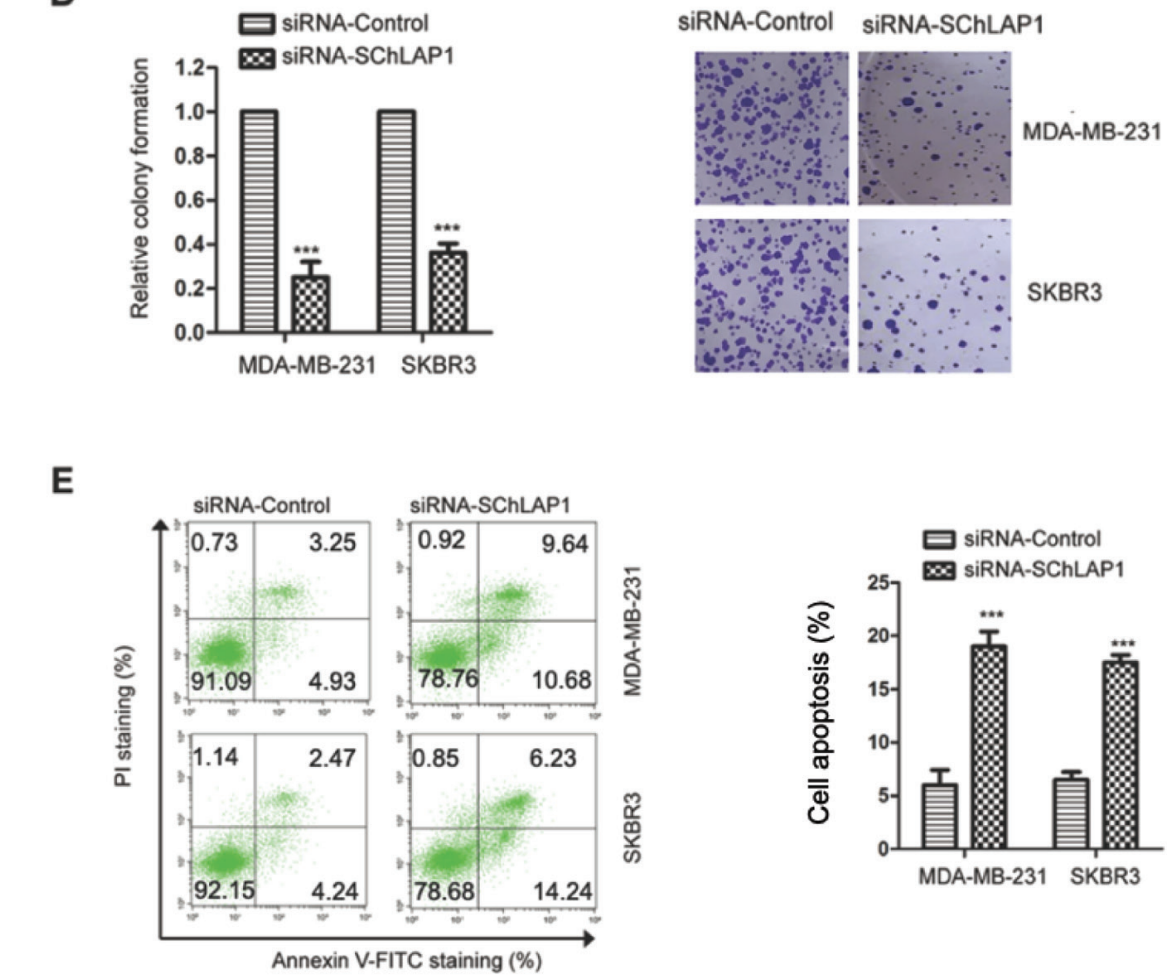

Figure 2. Knockdown of SChLAP1 inhibits the growth of triple negative breast cancer cells. (A) MDA-MB-231 and SKBR3 cells were transfected with control-siRNA or SChLAP1-siRNA. The knockdown of SChLAP1 was confirmed via reverse transcription-quantitative PCR analysis. A Cell Counting Kit-8 assay was performed to determine the viability of (B) MDA-MB-231 and (C) SKBR3 cells after transfection with siRNA-SChLAP1. (D) Colony formation ability was suppressed in MDA-MB-231 and SKBR3 cells upon knockdown of SChLAP1 (magnification, x40). (E) Knockdown of SChLAP1 significantly promoted the apoptosis of both MDA-MB-231 and SKBR3 cells compared with cells expressing a control vector. ${ }^{* * *} \mathrm{P}<0.001$ vs. siRNA-Control. OD, optical density; siRNA, small interfering RNA; SChLAP1, second chromosome locus associated with prostate-1.

compared with that of the adjacent normal tissues (Fig. 3E). Moreover, Pearson's correlation analysis indicated that the expression level of miR-524-5p in TNBC tissues was moderately, negatively correlated with SChLAP1 expression (Fig. 3F). These results demonstrated that SChLAP1 sponged miR-524-5p and negatively regulated the expression of miR-524-5p in TNBC cells.

HMGA2 is a target of miR-524-5p in TNBC. As miR-524-5p was identified as a target of SChLAP1, to further understand the importance of miR-524-5p in mediating the function of SChLAP1, the effects of miR-524-5p on the viability of TNBC cells were determined by transfecting miR-524-5p mimic into both MDA-MB-231 and SKBR3 cells. The overexpression of miR-524-5p was confirmed via RT-qPCR (Fig. 4A). The CCK-8 assay demonstrated that overexpression of miR-524-5p significantly inhibited the viability of both MDA-MB-231 and SKBR3 cells (Fig. 4B and C), which suggested that miR-524-5p had suppressive effects, and was consistent with the downregulated expression of miR-524-5p in TNBC.

To investigate the functional mechanism of miR-524-5p in TNBC, the downstream targets of miR-524-5p were also predicted using the online TargetScan database. It was determined that HMGA2 was a functional target of miR-524-5p, as the 3'-UTR of HMGA2 harbored the complementary binding sites of miR-524-5p (Fig. 4D). To validate this hypothesis, the WT or corresponding Mut 3'-UTR of HMGA2 was inserted into the luciferase vector. Both MDA-MB-231 and SKBR3 cells were co-transfected with miR-524-5p mimic and luciferase plasmid expressing the WT or Mut 3'-UTR of HMGA2. The results demonstrated that the luciferase intensity was significantly suppressed by miR-524-5p in WT group, but not by the Mut 3'-UTR of HMGA2 (Fig. 4E and F). 
A

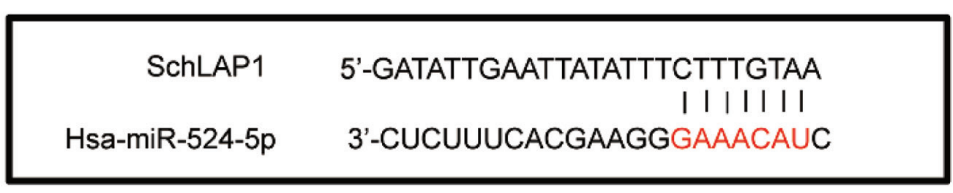

B

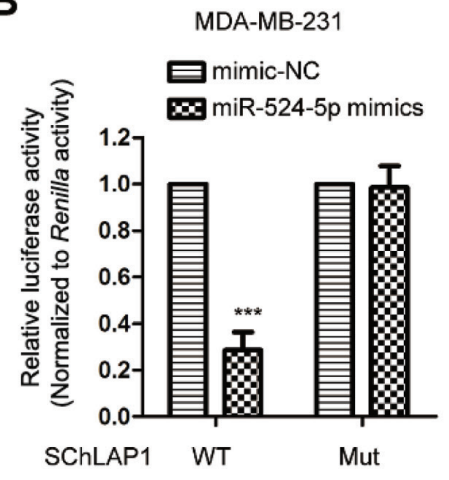

E

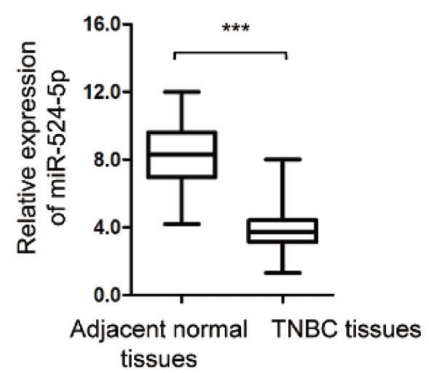

C

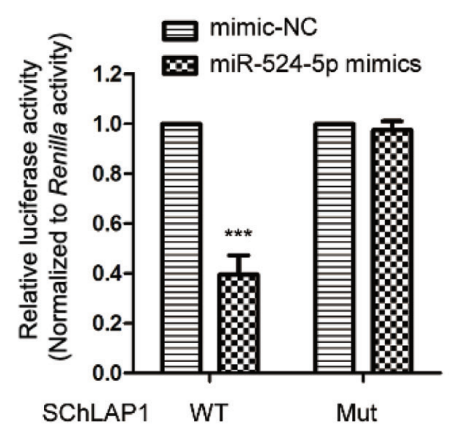

D

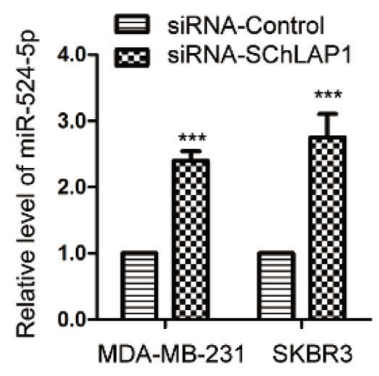

$\mathbf{F}$

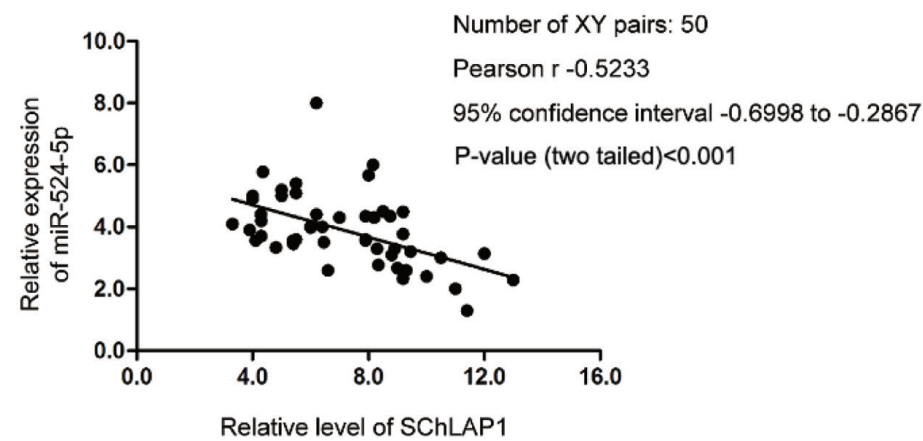

Figure 3. SChLAP1 acts as a sponge of miR-524-5p. (A) Predicted binding site of miR-524-5p on the sequence of SChLAP1. Luciferase activity of (B) MDA-MB-231 and (C) SKBR3 cells co-transfected with miR-524-5p mimics and WT-SChLAP1 was significantly decreased, while the activity of cells expressing miR-524-5p and Mut-SChLAP1 was not obviously affected. (D) Knockdown of SChLAP1 increased the expression of miR-524-5p in MDA-MB-231 and SKBR3 cells. (E) Expression of miR-524-5p in TNBC tissues was significantly downregulated compared with that of adjacent non-cancerous tissues. (F) Expression level of miR-524-5p was negatively correlated with SChLAP1 expression in TNBC tissues. ${ }^{* * *} \mathrm{P}<0.001 \mathrm{vs}$. corresponding control. NC, negative control; miR, microRNA; WT, wild-type; Mut, mutant; TNBC, triple negative breast cancer; siRNA, small interfering RNA; SChLAP1, second chromosome locus associated with prostate-1.

To further confirm that HMGA2 was a target of miR-524-5p, the mRNA and protein expression levels of HMGA2 were detected via RT-qPCR and western blotting, respectively. The data identified that HMGA2 expression was significantly decreased after the transfection of miR-524-5p (Fig. 4G and $\mathrm{H}$ ). These results demonstrated HMGA2 was a functional target of miR-524-5p in TNBC.

A SChLAP1/miR-524-5p/HMGA2 regulatory pathway is critical for the proliferation of TNBC cells. To investigate the regulation of SChLAP1 on the expression of HMGA2, the mRNA and protein expression levels of HMGA2 in MDA-MB-231 and SKBR3 cells were detected after the knockdown of SChLAP1. As presented in Fig. 5A and B, knockdown of SChLAP1 significantly decreased both the mRNA and protein expression levels of HMGA2 in TNBC cells.

To determine whether SChLAP1 exerted its role via the miR-524-5p/HMGA2 pathway, rescue experiments were performed by transfecting HMGA2. The expression of transfected Flag-tagged HMGA2 was validated via western blotting using anti-Flag antibody (Fig. 5C). The CCK-8 assay demonstrated that knockdown of SChLAP1 suppressed the viability of TNBC cells, while overexpression of HMGA2 significantly rescued the viability of SChLAP1-knockdown TNBC cells (Fig. 5D and E). Consistently, overexpression of HMGA2 also attenuated the suppressed colony formation capacity of TNBC cells that was induced by SChLAP1 knockdown (Fig. 5F). These findings demonstrated the critical function of the miR-524-5p/HMGA2 pathway in mediating the role of SChLAP1 in the malignancy of TNBC.

\section{Discussion}

Multiple lncRNAs have been identified as oncogenes or tumor suppressors that can regulate the progression of different human cancer types, including TNBC $(12,13)$. Based on the sequencing data, the expression of SChLAP1 was significantly decreased in TNBC tissues compared with that of the non-cancerous tissues, ranking top 10 among all the genes. Aberrant expression of SChLAP1 has been reported to serve an oncogenic role in the development of glioblastoma (26) and prostate cancer $(19,20)$. Increased expression levels of 
A

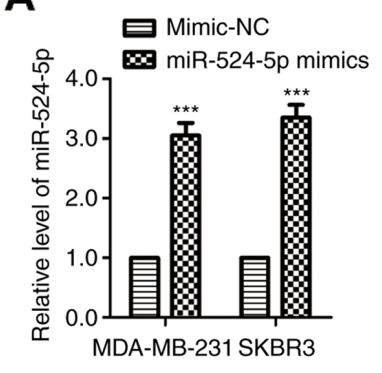

B

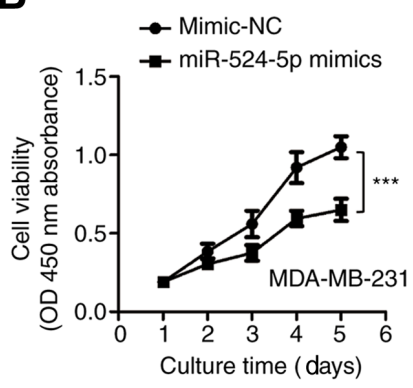

C

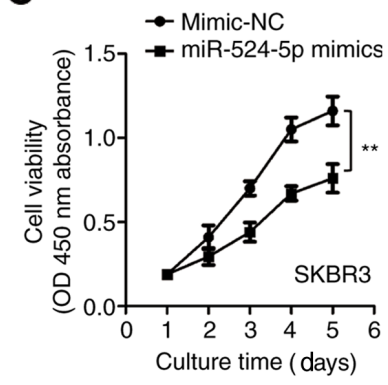

D

Position of 195-202 of HMGA2 3'-UTR 5'-UAUAUUAAUCACCUUCUUUGUAA

| | | || | |

Hsa-miR-524-5p 3'-CUCUUUCACGAAGGGAAACAUC

E

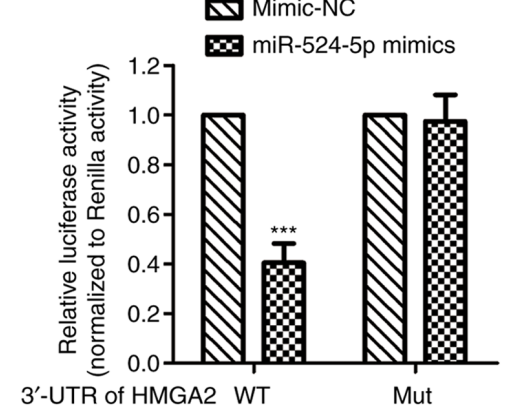

G

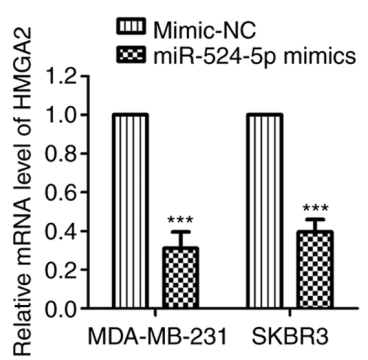

$\mathbf{F}$

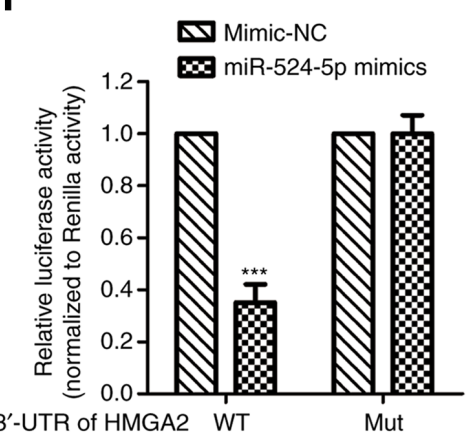

H

Mimic-NC miR-524-5p mimics

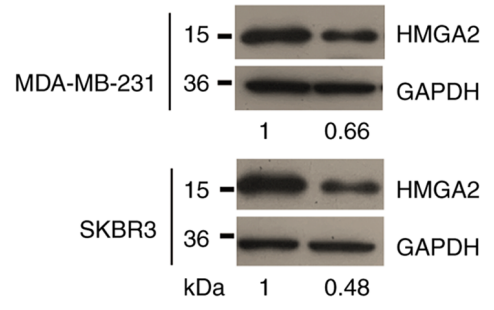

Figure 4. HMGA2 is a target of miR-524-5p in triple negative breast cancer. (A) MDA-MB-231 and SKBR3 cells were transfected with miR-524-5p mimic and mimic-NC, the expression of miR-524-5p was validated via reverse transcription-quantitative PCR. Cell Counting Kit-8 assay showed the decreased viability of (B) MDA-MB-231 and (C) SKBR3 cells induced by miR-524-5p overexpression. (D) Predicted binding sites of miR-524-5p in the 3'-UTR of HMGA2. Relative luciferase activities in (E) MB-231 and (F) SKBR3 cells co-transfected with miR-524-5p mimics or mimic-NC and luciferase reporter plasmid containing WT or Mut 3'-UTR of HMGA2. Overexpression of miR-524-5p decreased the (G) mRNA and (H) protein expression levels of HMGA2 in TNBC cells. The protein expression level of HMGA2 was semi-quantified by normalizing the level of GAPDH using ImageJ software. ${ }^{* *} \mathrm{P}<0.01{ }^{* * * *} \mathrm{P}<0.001$ vs. mimic-NC. UTR, untranslated region; NC, negative control; miR, microRNA; WT, wild-type; Mut, mutant; HMGA2, High Mobility Group AT-Hook 2; OD, optical density.

SChLAP1 in prostate cancer tissues may serve as a biomarker for the prognosis of patients with cancer (18). Overexpressed SChLAP1 promotes the proliferation and metastasis of cancer cells, and predicts poor outcomes of patients with cancer (19). These findings suggested that targeting SChLAP1 may be a potential strategy to disrupt cancer progression; however, the role of SChLAP1 in TNBC remains poorly understood.

The present results indicated that SChLAP1 was upregulated and associated with lymph node metastasis of patients with TNBC. The functional analysis revealed that knockdown of SChLAP1 significantly inhibited the viability and colony formation, as well as induced the apoptosis of TNBC cells. These findings demonstrated the essential role of SChLAP1 in the progression of TNBC. However, further study will be necessary to evaluate the correlation of SChLAP1 with the clinical parameters of patients with TNBC to determine whether aberrant expression of SChLAP1 can be used as an independent biomarker for the prognosis of TNBC. In addition to SChLAP1, those genes that are differentially expressed in TNBC also warrant further investigation to identify their role in the development of TNBC.

Increasing evidence has revealed that lncRNAs exert their functions in cancer by acting as ceRNAs to sponge miRNA and regulate the expression of miRNAs (27-29). A recent study reported that SChLAP1 accelerated the malignant phenotype of prostate cancer by sponging miR-198 and activating MAPK1 signaling (20). In the current study, the potential miRNAs capable of binding to SChLAP1 were predicted using the online tool. Both the prediction and the luciferase reporter assay 
A

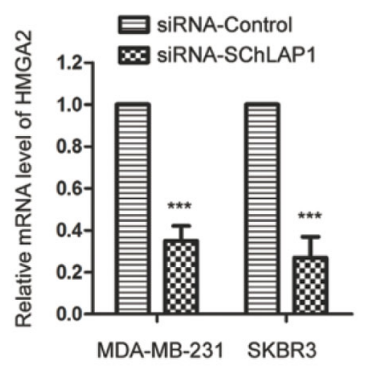

B

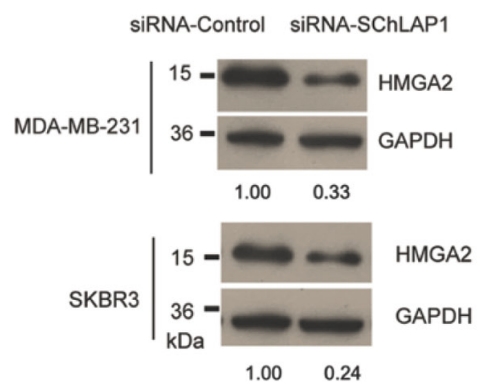

C

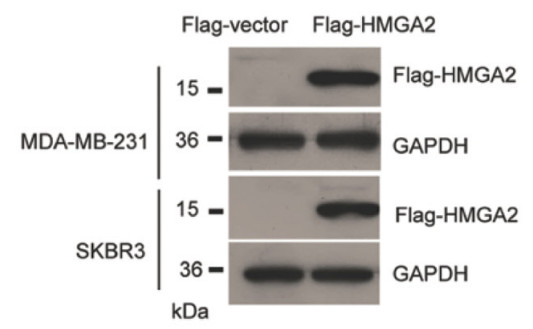

D

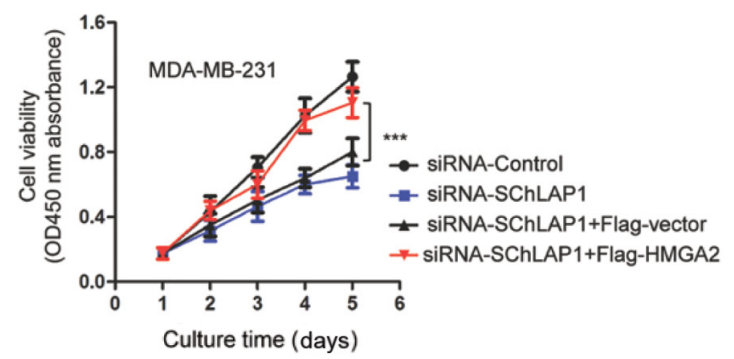

E

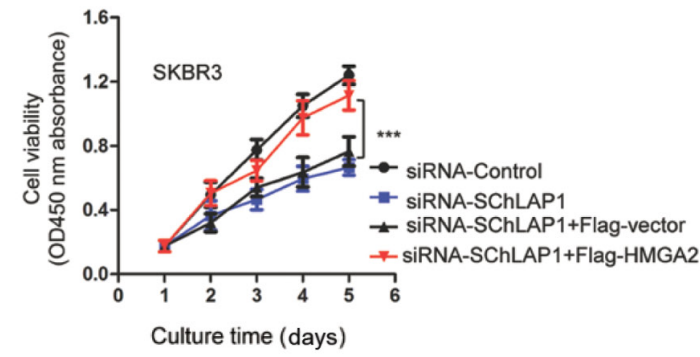

F

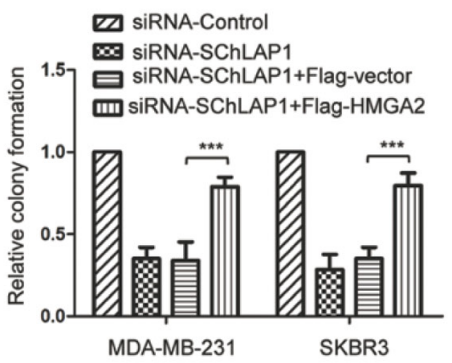

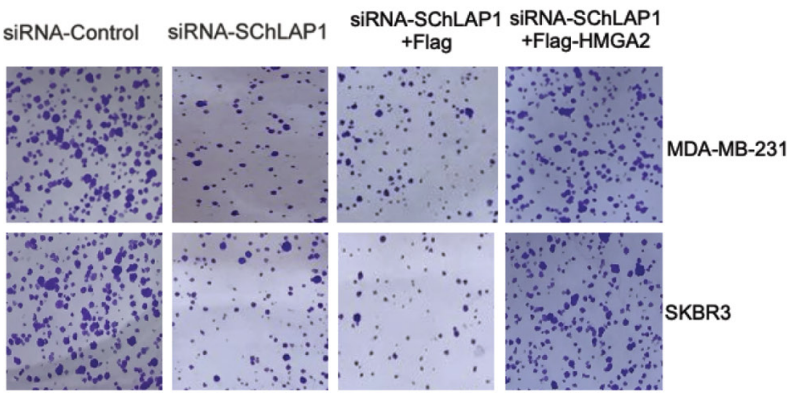

Figure 5. Reintroduction of HMGA2 reverses SChLAP1 knockdown-induced viability defects in TNBC cells. Knockdown of SChLAP1 decreased both the (A) mRNA and (B) protein expression levels of HMGA2 in MDA-MB-231 and SKBR3 cells. The protein expression level of HMGA2 was semi-quantified by normalizing the level of GAPDH using the ImageJ software. (C) Expression of Flag-tagged HMGA2 was detected via western blotting with anti-Flag antibody. Overexpression of HMGA2 significantly attenuated the effect of SChLAP1 knockdown on the viability of (D) MDA-MB-231 and (E) SKBR3 cells. (F) Co-transfection of HMGA2 promoted the colony formation of TNBC cells compared with cells expressing siRNA-SChLAP1 (magnification, x40). ${ }^{* * *} \mathrm{P}<0.001$ vs. control group. HMGA2, High Mobility Group AT-Hook 2; TNBC, triple negative breast cancer; siRNA, small interfering RNA; SChLAP1, second chromosome locus associated with prostate-1; OD, optical density.

validated the binding between SChLAP1 and miR-524-5p. Interestingly, miR-524-5p exhibits a low expression level and acts as a tumor suppressor in multiple cancer types, including gastric cancer, glioma and papillary thyroid carcinoma (30-34).

In the present study, knockdown of SChLAP1 increased the expression of miR-524-5p in TNBC cells. Consistent with the upregulation of SChLAP1 in TNBC, miR-524-5p expression was downregulated and negatively correlated with that of SChLAP1 in TNBC tissues. Additionally, overexpression of miR-524-5p suppressed the viability of TNBC cells, suggesting that miR-524-5p exerts a tumor-suppressive role in TNBC. However, it is necessary to investigate the clinical significance of abnormally expressed miR-524-5p and validate the inhibitory function of miR-524-5p in TNBC using in vivo experiments.

HMGA2 is a member of the high motility group protein family and can bind the DNA minor groove at sequences rich with $\mathrm{A}$ and $\mathrm{T}$ nucleotides to activate gene transcription (35). Accumulating evidence has indicated that there was increased expression of HMGA2 in different human cancer types, including pancreatic cancer and gastric cancer, and that it was involved in the epithelial-to-mesenchymal transition of multiple epithelial carcinomas (36). Overexpression of HMGA2 is strongly associated with the poor prognosis of patients with colorectal cancer (37). HMGA2 has also been identified as a target of miRNAs that modulates the development of cancer (38-42). For example, miR-9 exerted anti-cancer effects in hepatocellular carcinoma by targeting HMGA2 (43), while miR-204-3p decreased HMGA2 and repressed the malignant phenotype of colon cancer (44).

In the current study, it was found that miR-524-5p bound to the 3'-UTR of HMGA2 and inhibited the expression of HMGA2 in TNBC cells. Consistent with the increased expression levels of miR-524-5p, knockdown of SchLAP1 suppressed both the mRNA and protein expression levels of HMGA2 in TNBC cells. The rescue experiments demonstrated that overexpression of HMGA2 attenuated the reduced growth of TNBC cells by downregulating SChLAP1. These findings indicate the critical function of the miR-524-5p/HMGA2 axis in the oncogenic role of SChLAP1 in TNBC. 
In conclusion, the present study demonstrated that SChLAP1 was upregulated and positively associated with the advanced progression of TNBC. The results suggested that SChLAP1 regulates the malignant behaviors of TNBC cells by sponging miR-524-5p. This evidence provides a novel perspective on the molecular mechanism of SChLAP1 in the progression of TNBC.

\section{Acknowledgements}

Not applicable.

\section{Funding}

No funding was received.

\section{Availability of data and materials}

The datasets used and/or analyzed during the present study are available from the corresponding author upon reasonable request.

\section{Authors' contributions}

$\mathrm{XB}, \mathrm{HZ}$ and $\mathrm{JX}$ designed the study. XB performed the experiments. SZ and JQ collected the tissue samples and analyzed gene expression via reverse transcription-quantitative PCR. $\mathrm{XX}$ and WL performed the dual-luciferase reporter assay and western blotting. XB, HZ and JX wrote the manuscript. HZ and JX confirmed the authenticity of all raw data. All authors read and approved the final manuscript.

\section{Ethics approval and consent to participate}

This work was approved by the Ethics Committee of Shanxi Provincial Cancer Hospital. All patients provided written informed consent prior to the study.

\section{Patient consent for publication}

Not applicable.

\section{Competing interests}

The authors declare that they have no competing interests.

\section{References}

1. Medina MA, Oza G, Sharma A, Arriaga LG, Hernandez Hernandez JM, Rotello VM and Ramirez JT: Triple-negative breast cancer: A review of conventional and advanced therapeutic strategies. Int J Environ Res Public Health 17: 2078, 2020

2. da Silva JL, Cardoso Nunes NC, Izetti P, de Mesquita GG and de Melo AC: Triple negative breast cancer: A thorough review of biomarkers. Crit Rev Oncol Hematol 145: 102855, 2020.

3. Pandy JGP, Balolong-Garcia JC, Cruz-Ordinario MVB and Que FVF: Triple negative breast cancer and platinum-based systemic treatment: A meta-analysis and systematic review. BMC Cancer 19: 1065, 2019.

4. De Laurentiis M, Cianniello D, Caputo R, Stanzione B, Arpino G, Cinieri S, Lorusso V and De Placido S: Treatment of triple negative breast cancer (TNBC): Current options and future perspectives. Cancer Treat Rev 36 (Suppl 3): S80-S86, 2010.
5. Wang KC and Chang HY: Molecular mechanisms of long noncoding RNAs. Mol Cell 43: 904-914, 2011.

6. Mercer TR, Dinger ME and Mattick JS: Long non-coding RNAs: Insights into functions. Nat Rev Genet 10: 155-159, 2009.

7. Fabian MR, Sonenberg N and Filipowicz W: Regulation of mRNA translation and stability by microRNAs. Annu Rev Biochem 79: 351-379, 2010.

8. Mohr AM and Mott JL: Overview of microRNA biology. Semin Liver Dis 35: 3-11, 2015.

9. Bartel DP: MicroRNAs: Genomics, biogenesis, mechanism, and function. Cell 116: 281-297, 2004.

10. Lu TX and Rothenberg ME: MicroRNA. J Allergy Clin Immunol 141: 1202-1207, 2018.

11. Rodriguez Bautista R, Ortega Gómez A, Hidalgo Miranda A, Zentella Dehesa A, Villarreal-Garza C, Ávila-Moreno F and Arrieta O: Long non-coding RNAs: Implications in targeted diagnoses, prognosis, and improved therapeutic strategies in human non- and triple-negative breast cancer. Clin Epigenetics 10: 88 , 2018.

12. Wang Q, Gao S, Li H, Lv M and Lu C: Long noncoding RNAs (lncRNAs) in triple negative breast cancer. J Cell Physiol 232: 3226-3233, 2017.

13. Kong X, Liu W and Kong Y: Roles and expression profiles of long non-coding RNAs in triple-negative breast cancers. J Cell Mol Med 22: 390-394, 2018.

14. Piasecka D, Braun M, Kordek R, Sadej R and Romanska H: MicroRNAs in regulation of triple-negative breast cancer progression. J Cancer Res Clin Oncol 144: 1401-1411, 2018.

15. Naorem LD, Prakash VS, Muthaiyan M and Venkatesan A: Comprehensive analysis of dysregulated lncRNAs and their competing endogenous RNA network in triple-negative breast cancer. Int J Biol Macromol 145: 429-436, 2020.

16. Xu ST, Xu JH, Zheng ZR, Zhao QQ, Zeng XS, Cheng SX, Liang YH and Hu QF: Long non-coding RNA ANRIL promotes carcinogenesis via sponging miR-199a in triple-negative breast cancer. Biomed Pharmacother 96: 14-21, 2017.

17. Fan H, Yuan J, Li X, Ma Y, Wang X, Xu B and Li X: LncRNA LINC00173 enhances triple-negative breast cancer progression by suppressing miR-490-3p expression. Biomed Pharmacother 125: $109987,2020$.

18. Mehra R, Udager AM, Ahearn TU, Cao X, Feng FY, Loda M, Petimar JS, Kantoff P, Mucci LA and Chinnaiyan AM: Overexpression of the Long Non-coding RNA SChLAP1 independently predicts lethal prostate cancer. Eur Urol 70: 549-552, 2016.

19. Prensner JR, Iyer MK, Sahu A, Asangani IA, Cao Q, Patel L, Vergara IA, Davicioni E, Erho N, Ghadessi M, et al: The long noncoding RNA SChLAP1 promotes aggressive prostate cancer and antagonizes the SWI/SNF complex. Nat Genet 45: 1392-1398, 2013.

20. Li Y, Luo H, Xiao N, Duan J, Wang Z and Wang S: Long noncoding RNA SChLAP1 accelerates the proliferation and metastasis of prostate cancer via targeting miR-198 and promoting the MAPK1 pathway. Oncol Res 26: 131-143, 2018.

21. Zhang J, Shi Z, Nan Y and Li M: Inhibiting malignant phenotypes of the bladder cancer cells by silencing long noncoding RNA SChLAP1. Int Urol Nephrol 48: 711-716, 2016.

22. Livak KJ and Schmittgen TD: Analysis of relative gene expression data using real-time quantitative PCR and the 2(-Delta Delta C(T)) method. Methods 25: 402-408, 2001.

23. Dobin A, Davis CA, Schlesinger F, Drenkow J, Zaleski C, Jha S, Batut P, Chaisson M and Gingeras TR: STAR: Ultrafast universal RNA-seq aligner. Bioinformatics 29: 15-21, 2013.

24. Liao Y, Smyth GK and Shi W: featureCounts: An efficient general purpose program for assigning sequence reads to genomic features. Bioinformatics 30: 923-930, 2014.

25. Chen Y and Wang X: miRDB: An online database for prediction of functional microRNA targets. Nucleic Acids Res 48: D127-D131, 2020.

26. Ji J, Xu R, Ding K, Bao G, Zhang X, Huang B, Wang X, Martinez A, Wang X, Li G, et al: Long Noncoding RNA SChLAP1 forms a growth-promoting complex with HNRNPL in human glioblastoma through stabilization of ACTN4 and activa-

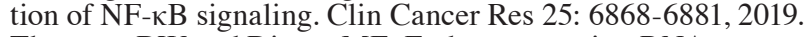

27. Thomson DW and Dinger ME: Endogenous microRNA sponges: Evidence and controversy. Nat Rev Genet 17: 272-283, 2016.

28. Chan JJ and Tay Y: Noncoding RNA: RNA regulatory networks in cancer. Int J Mol Sci 19: 310, 2018.

29. Tay Y, Rinn J and Pandolfi PP: The multilayered complexity of ceRNA crosstalk and competition. Nature 505: 344-352, 2014. 
30. Liu H, Chen $X$, Lin $T$, Chen $X$, Yan J and Jiang $S$ : MicroRNA-524-5p suppresses the progression of papillary thyroid carcinoma cells via targeting on FOXE1 and ITGA3 in cell autophagy and cycling pathways. J Cell Physiol 234: 18382-18391, 2019.

31. Liu GH, Liu YH, Yang Z, Zhu AL and Zhao CL: MicroRNA-524-5p suppresses the growth and invasive abilities of gastric cancer cells. Oncol Lett 11: 1926-1932, 2016.

32. Zhu CY, Meng FQ and Liu J: MicroRNA-524-5p suppresses cell proliferation and promotes cell apoptosis in gastric cancer by regulating CASP3. Eur Rev Med Pharmacol Sci 23: 7968-7977, 2019.

33. Zhen W, Qiu D, Zhiyong C, Xin W, Mengyao J, Dimin Z, Chonghui H, Haijun W and Yonghong Z: MicroRNA-524-5p Functions as a tumor suppressor in a human pituitary tumor-derived cell line. Horm Metab Res 49: 550-557, 2017.

34. Zhao K, Wang Q, Wang Y, Huang K, Yang C, Li Y, Yi K and Kang C: EGFR/c-myc axis regulates TGF $/$ Hippo/Notch pathway via epigenetic silencing miR-524 in gliomas. Cancer Lett 406: 12-21, 2017

35. Zhang S, Mo Q and Wang X: Oncological role of HMGA2 (Review). Int J Oncol 55: 775-788, 2019.

36. De Martino M, Fusco A and Esposito F: HMGA and Cancer: A review on patent literatures. Recent Pat Anticancer Drug Discov 14: 258-267, 2019.

37. Huang YM, Cheng CH, Pan SL, Yang PM, Lin DY and Lee KH: Gene expression signature-based approach identifies antifungal drug ciclopirox as a novel inhibitor of HMGA2 in colorectal cancer. Biomolecules 9: 688, 2019.
38. Kang NN, Ge SL, Zhang RQ, Huang YL, Liu SD and Wu KM: MiR-490-3p inhibited the proliferation and metastasis of esophageal squamous cell carcinoma by targeting HMGA2. Eur Rev Med Pharmacol Sci 22: 8298-8305, 2018.

39. Xing F, Song Z and He Y: MiR-219-5p inhibits growth and metastasis of ovarian cancer cells by targeting HMGA2. Biol Res 51: 50, 2018.

40. Sun J, Qiao Y, Song T and Wang H: MiR495 suppresses cell proliferation by directly targeting HMGA2 in lung cancer. Mol Med Rep 19: 1463-1470, 2019.

41. Wang MJ, Zhang H, Li J and Zhao HD: microRNA-98 inhibits the proliferation, invasion, migration and promotes apoptosis of breast cancer cells by binding to HMGA2. Biosci Rep 38: BSR 20180571, 2018.

42. Wang J, Liang H, Ge H, Guo X, Gu D and Yuan Y: MicroRNA3633p inhibits hepatocarcinogenesis by targeting HMGA2 and is associated with liver cancer stage. Mol Med Rep 19: 935-942, 2019.

43. Xu X, Zou H, Luo L, Wang X and Wang G: MicroRNA-9 exerts antitumor effects on hepatocellular carcinoma progression by targeting HMGA2. FEBS Open Bio 9: 1784-1797, 2019.

44. Xi X, Teng M, Zhang L, Xia L, Chen J and Cui Z: MicroRNA-204-3p represses colon cancer cells proliferation, migration, and invasion by targeting HMGA2. J Cell Physiol 235: $1330-1338,2020$

(i) (9) This work is licensed under a Creative Commons

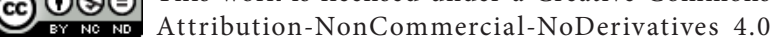
International (CC BY-NC-ND 4.0) License. 